\title{
FIGO Stage IA1
}

National Cancer Institute

\section{Source}

National Cancer Institute. FlGO Stage IA1. NCI Thesaurus. Code C96246.

A FIGO stage term that applies to gynecologic cancers. For cervical cancer, it refers to invasive cancer that is diagnosed microscopically only. The stromal invasion has a maximum depth of $3.00 \mathrm{~mm}$ and a horizontal spread of $7.00 \mathrm{~mm}$ or less; there is no FIGO stage IA1 for endometrial cancer. 